\title{
Successful Autotransplantation of an Immature Third Molar- A Case Report
}

\author{
Dr Kavita Dube ${ }^{1}$, Dr Bonny Paul ${ }^{2}$,Dr Abhilash Shankaran ${ }^{3}$, Dr Abhishek \\ Sharma $^{3}$ \\ 1(Reader,Department of Endodontics.Hitkarini Dental College \& hospital ,Jabalpur.India) \\ 2(Professor,Department of Endodontics Hitkarini Dental College \& hospital,Jabalpur.India) \\ 3.(Post graduate, Department of Endodontics Hitkarini Dental College \& hospital, Jabalpur.India)
}

\begin{abstract}
This case report describes the autotransplantation of a third molar with developing roots in a young female patient. Following transplantation into the socket of mandibular first molar, the tooth was stabilized with sutures and a custom made semi rigid splint. On recall examination one year after completion of the surgical technique, the transplant responded positively to electric pulp testing and showed absence of infection, ankylosis or progressive resorption. The transplantation of a third molar can be seen as a promising method to replace a lost permanent tooth.

Clinical relevance :The permanent first molar is the most caries prone permanent tooth, probably because of its early exposure to the oral environment. Unrestorable teeth need to be extracted. Its extraction leads to unfavorable occlusal changes. Fixed prosthesis and implants are not feasible in growing patients because they may impede the normal growth of facial bones, in particular, of the alveolar process. This case report describes autotransplantation of third molar with incompletely formed roots as a viable treatment option when permanent first molar needs to be extracted. The use of semirigid splint has been demonstrated and follow up showed good prognosis.
\end{abstract}

Keywords: third molar, autotransplantation, semi rigid splint

\section{Introduction}

Unilateral first molar extractions cause dental midline deviations in both arches, being more prominent in the mandibular arch. Unilateral first molar extraction during growth and development can result in remarkable skeletal asymmetry, especially in the lower third of face. .[1]

Auto transplantation is a viable option for the treatment of a missing tooth when a suitable donor tooth is available. Successful tooth auto transplantation offers improved aesthetics, arch form, dentofacial development, mastication, speech and arch integrity. In addition, it maintains natural spacing, preventing migration of adjacent and opposing teeth. It also maintains the morphology of the alveolar ridge through proprioceptive stimulation.[2]

Autotransplantation was first described by Apfel when immature third molars were used to replace decayed first molars .[3] Following transplantation, the fixation period and method are known to influence the treatment outcome. This case report describes the successful auto transplantation of an immature third molar in the alveolar socket of first molar followed by splinting with a semi-rigid acrylic splint.

\section{Case report.}

An eighteen year old female patient reported to the Department of Endodontics with the chief complaint of dull pain in her mandibular left first molar. A history of previous endodontic treatment was elicited which was carried out about six months ago.

Clinical examination showed the mandibular left first molar with extensive caries (Fig 1). Radiograph confirmed the history of previous endodontic treatment. The mesial root showed a separated instrument in the middle third and an associated periapical radiolucency .The distal canal showed a long separated fragment extending from the coronal third up to the junction of middle and apical third (Fig 2). 


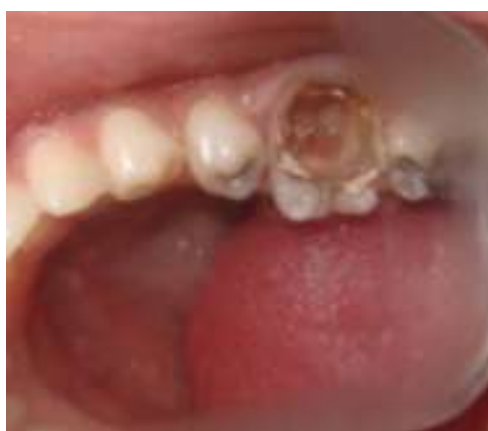

Figure 1 (pre operative photograph)

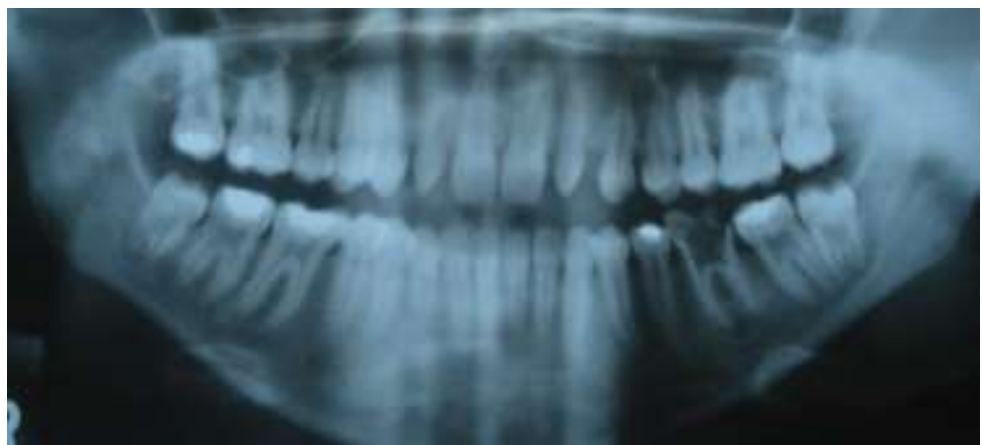

Figure 2 (pre operative radiograph)

Considering the poor prognosis for endodontic treatment, the young age of the patient and the involved treatment costs of alternative treatment modalities (implant), the patient was suggested a treatment protocol involving autotransplantation of the mandibular third molar after extraction of the tooth in question. The orthopantamograph showed the mandibular third molar, with developing roots suggestive of a suitable donor (Fig 2) .Patient was explained in detail the treatment plan and prognosis. An informed oral and written consent was obtained.

\section{Pre-operative procedure.}

Alginate impression of the mandibular arch was recorded and a cast in dental stone was made. A semi rigid splint was fabricated by adapting a $0.7 \mathrm{~mm}$ stainless steel wire around the right mandibular posterior teeth, starting from the mesial aspect of the first premolar to distal aspect of the second molar tooth (Fig 3). Acrylization was done using self cure clear acrylic. The splint was relieved from the tissue surface by trimming. The patient was prescribed broad spectrum antibiotics and chlorhexidine oral rinse two days before the operative procedure.

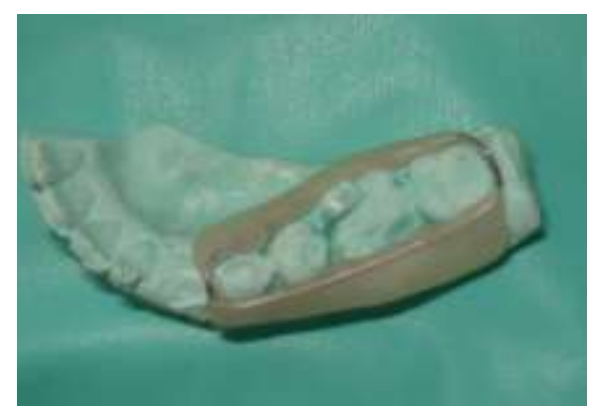

Fig 3 - flexible splint

\section{Operative procedure.}

After an oral rinse with $0.2 \%$ chlorhexidine, local anesthesia was administered (Lignocaine with $2 \%$ adrenaline) in the form of inferior alveolar nerve block. The mandibular first molar was extracted as atraumatically as possible, while taking care not to damage the walls of the alveolar socket. The intra-alveolar septum was trimmed with a rongeur, and the socket irrigated.

Following the extraction, a bayonet shaped incision was placed around the second molar, buccal and lingual mucoperiosteal flaps were raised using a periosteal elevator. Minimal amount of bone distal to the third molar was removed using a rose head bur in a slow speed hand piece using saline as coolant. The tooth was delivered atraumatically using a third molar forceps taking care not to damage the periodontal ligament.

The third molar was now held with a forceps above the CEJ taking care not to touch the root surface, thus preventing any damage to the periodontal ligament. It was carefully positioned into the alveolar socket of the first molar which it snugly fitted into, thus negating the need of any further alteration of the socket. The tooth was slightly depressible in the socket and remained in infraocclusion. It was stabilized by sutures placed interdentally and further stabilized by placing the custom made splint fabricated earlier. (Fig 4, 5) The patient was advised to eat soft diet for few days. 


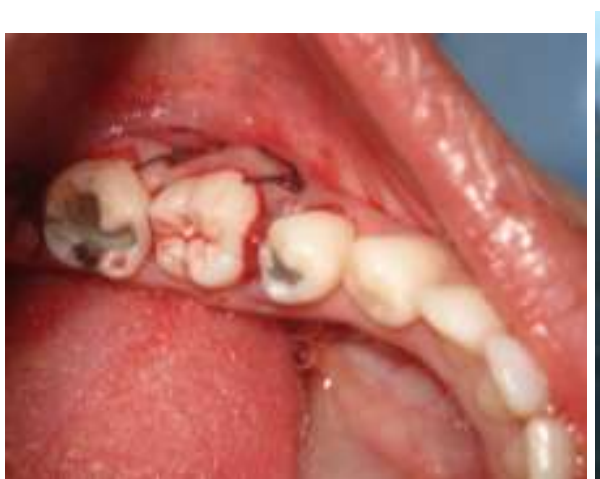

Fig 4- post operative photograph

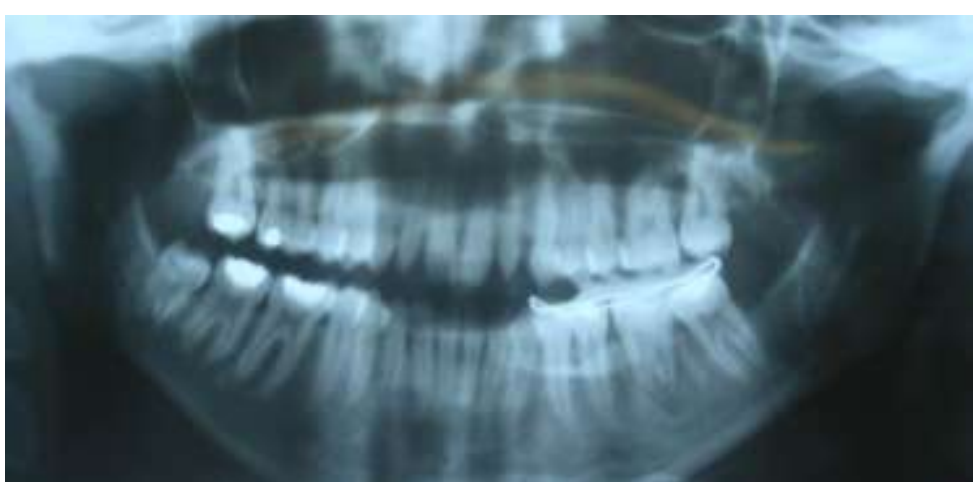

Fig 5- post operative radiograph

The post - operative period was uneventful. On the fifth post operative day the splint was removed and on the seventh post operative day, the sutures were removed. The patient was asymptomatic, although the tooth remained depressible in the socket. The patient was regularly followed on monthly basis for six months followed by bi-monthly check-up for a year. The radiograph recorded one year post operatively showed signs of developing root, also the tooth responded positively to electric pulp testing suggestive of favorable prognosis. (Fig 6)

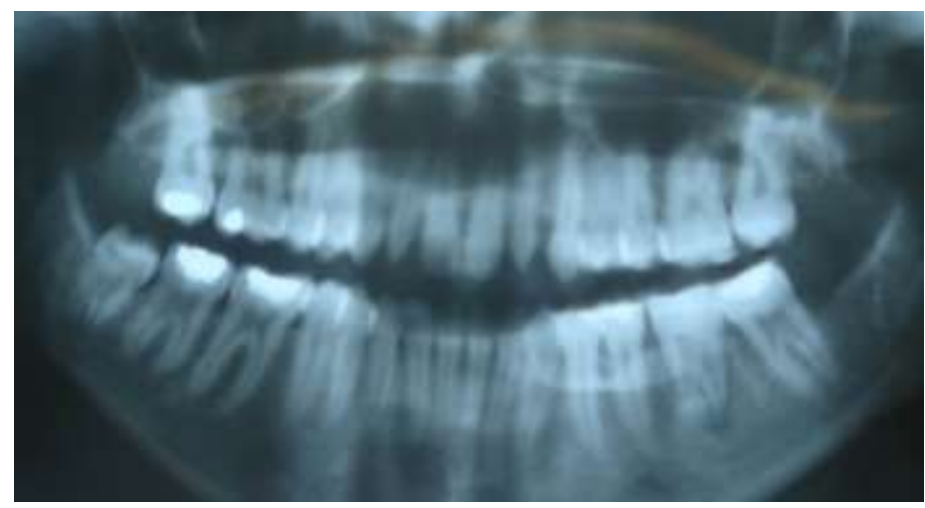

Fig 6- radiograph: 12 months follow up

\section{Discussion}

Premature loss of the first molar tooth results in mesial movements of the posterior teeth, the resultant loss of space, and over eruption of opposing teeth and consequent changes in the occlusion which must be avoided. Fixed prosthesis and implants are not feasible in growing patients because they may impede the normal growth of facial bones, in particular, of the alveolar process. Transplantation of an immature third molar with incompletely formed roots could possibly serve as a suitable treatment option as it helps to maintain alveolar bone and enables endosseous implantation without requiring bone regeneration .[4] It has been reported that during growth, a successful transplant preserves the alveolar bone, diminishes the extent of resorption of newly formed alveolar bone and provides functional stimulation.[5,6]

Many factors have been implicated to affect the prognosis of an autologous implant. Transplantation of teeth with immature roots offers higher success rates. This is attributed to the unimpeded root development of the transplant as well as the growth of the adjacent alveolar bone .[7] Transplanted teeth with incomplete root formation have $96 \%$ rate of pulp healing when compared to $15 \%$ for teeth with complete root formation .[8] Teeth with an apical diameter greater than one $\mathrm{mm}$ have a diminished risk of pulp necrosis because post operative revascularization is more likely.[9]

Different data have been published on the influence of splinting method and the period of fixation on the success of transplant. Following rigid or extended fixation, increased incidences of ankylosis and disturbances of pulp revascularization have been reported .[10] The splint should not force the tooth against the bony walls of the alveolus because it may damage the periodontium .[11] Most reports suggest flexible splinting. The splint used in this case permitted some functional movement of the transplant, thus would have helped in stimulating periodontal ligament cell activity and repair.

The absence of transplant mobility, pain, root resorption in addition to positive pulp vitality test and continued root formation one year post operatively were suggestive of favorable prognosis, although long term success rate needs to be evaluated. 


\section{Conclusion:}

The success of this case can be attributed to the atraumatic surgical technique, the immature stage of transplant and flexible splinting. Whenever unrestorable teeth need to be extracted, tooth transplantation could serve as a viable alternative rather than replacing the tooth with a fixed or removable prosthesis. Clinicians should make the patient aware of this alternate treatment modality and its probable prognosis.

\section{References:}

[1] Cağlaroğlu M, Kilic N, Erdem A. Effects of early unilateral first molar extraction on skeletal asymmetry. Am J Orthod Dentofacial Orthop 2008; 134:270-5.

[2] Hernandez SL, Cuestas-Carnero R. Autogenic tooth transplantation: a report of ten cases. J Oral Maxillofac Surg 1988; 46:1051-5.

[3] Apfel H. Autoplasty of enucleated prefunctional third molars. J Oral Surg 1950; 8:289-96.

[4] Cohen AS, Shen TC, Pogrel MA. Transplanting teeth successfully: autografts and allografts that work. J Am Dent Assoc 1995; 126:481-5.

[5] Hillerup S, Dahl E, Schwartz O, Hjørting-Hansen E. Tooth transplantation to bone graft in cleft alveolus. Cleft Palate J 1987; 24:137-41.

[6] Kvint S, Lindsten R, Magnusson A, Nilsson P, Bjerklin K.Autotransplantation of Teeth in 215 Patients. A follow-up study. Angle Orthod 2010; 80:446-51.

[7] Mendes RA, Rocha G.Mandibular Third Molar Autotransplantation - Literature Review with Clinical cases. J Can Dent Assoc 2004; 70:761-6.

[8] Andreasen JO.Autotransplantation of molars.In: Atlas of replantation and transplantation of teeth. Philadelphia: WB Saunders Company $1992 ; 111-34$.

[9] Thomas S, Turner SR, Sandy JR. Autotransplantation of teeth: is there a role? Br J Orthod 1998; 25:275-82.

[10] Pogrel. Evaluation of over 400 autogenous tooth transplants. J Oral Maxillofac surg 1987;45:205-11.

[11] Pohl Y, Filippi A, Tekin U, Kirschner H. Periodontal healing after intentional auto-alloplastic reimplantation of injured immature upper front teeth. J Clin Periodontol 2000; 27:198-204. 